\title{
HYPERSURFACES OF ALMOST $r$-PARACONTACT RIEMANNIAN MANIFOLD ENDOWED WITH A QUARTER SYMMETRIC METRIC CONNECTION
}

\author{
Mobin Ahmad, Jae-Bok Jun, And Abdul Haseeb
}

\begin{abstract}
We define a quarter symmetric metric connection in an almost $r$-paracontact Riemannian manifold and we consider invariant, noninvariant and anti-invariant hypersurfaces of an almost $r$-paracontact Riemannian manifold endowed with a quarter symmetric metric connection.
\end{abstract}

\section{Introduction}

In [1], T. Adati studied Hypersurfaces of almost paracontact Riemannian manifolds. In [3], A. Bucki, considered hypersurfaces of almost $r$-paracontact Riemannian manifold. Some properties of invariant hypersurfaces of an almost $r$-paracontact Riemannian manifold were investigated in [4] by A. Bucki and A. Miernowski. In [2], M. Ahmad, C. Ozgur, and A. Haseeb studied hypersurfaces of almost $r$-paracontact Riemannian manifold with quarter symmetric non-metric connection. Moreover in [7], I. Mihai and K. Matsumoto studied submanifolds of an almost $r$-paracontact Riemannian manifold of $P$-Sasakian type.

Let $\nabla$ be a linear connection in an $n$-dimensional differentiable manifold $M$. The torsion tensor $T$ of $\nabla$ is given by

$$
T(X, Y)=\nabla_{X} Y-\nabla_{Y} X-[X, Y] .
$$

The connection $\nabla$ is symmetric if its torsion tensor $T$ vanishes, otherwise it is non-symmetric. The connection $\nabla$ is metric if there is a Riemannian metric $g$ in $M$ such that $\nabla g=0$, otherwise it is non-metric. It is well known that a linear connection is symmetric and metric if it is the Levi-Civita connection. In [6], S. Golab introduced the idea of a quarter symmetric linear connection if its torsion tensor $T$ is of the form

$$
T(X, Y)=u(Y) \phi X-u(X) \phi Y,
$$

Received June 4, 2008.

2000 Mathematics Subject Classification. 53C05, 53D12.

Key words and phrases. hypersurfaces, almost $r$-paracontact Riemannian manifold, quarter symmetric metric connection. 
where $u$ is a 1 -form and $\phi$ is a tensor field of the type $(1,1)$. In [8], R. S. Mishra and S. N. Pandey considered a quarter symmetric metric $F$-connection and studied some of its properties. In [8], [9] and [10], some kinds of quarter symmetric metric connection were studied.

In this paper, we study quarter symmetric metric connection in an almost $r$-paracontact Riemannian manifold. We consider invariant, non-invariant and anti-invariant hypersurfaces of almost $r$-paracontact Riemannian manifold endowed with a quarter symmetric metric connection.

The paper is organized as follows: In Section 2, we give a brief introduction about an almost $r$-paracontact Riemannian manifold. In Section 3, we show that the induced connection on a hypersurface of an almost $r$-paracontact Riemannian manifold with quarter symmetric metric connection with respect to the normal is also a quarter symmetric metric connection. We find the characteristic properties of invariant, non-invariant and anti-invariant hypersurfaces of almost $r$-paracontact Riemannian manifold endowed with a quarter symmetric metric connection.

\section{Preliminaries}

Let $M$ be an $n$-dimensional Riemannian manifold with a positive definite metric $g$. If there exist a tensor field $\phi$ of type $(1,1), r$ vector fields $\xi_{1}, \xi_{2}, \ldots, \xi_{r}$ $(n>r), r$ 1-forms $\eta^{1}, \eta^{2}, \ldots, \eta^{r}$ such that

$$
\begin{gathered}
\eta^{\alpha}\left(\xi_{\beta}\right)=\delta_{\beta}^{\alpha}, \alpha, \beta \in(r)=\{1,2,3, \ldots, r\}, \\
\phi^{2}(X)=X-\eta^{\alpha}(X) \xi_{\alpha}, \\
\eta^{\alpha}(X)=g\left(X, \xi_{\alpha}\right), \alpha \in(r), \\
g(\phi X, \phi Y)=g(X, Y)-\sum_{\alpha} \eta^{\alpha}(X) \eta^{\alpha}(Y),
\end{gathered}
$$

where $X$ and $Y$ are vector fields on $M$, then the structure $\sum=\left(\phi, \xi_{\alpha}, \eta^{\alpha}, g\right)_{\alpha \in(r)}$ is said to be an almost $r$-paracontact Riemannian structure and $M$ is an almost $r$-paracontact Riemannian manifold [3]. From (2.1) through (2.4), we have for $\alpha \in(r)$

$$
\begin{gathered}
\phi\left(\xi_{\alpha}\right)=0, \quad \eta^{\alpha} \circ \phi=0, \\
\Phi(X, Y)=g(\phi X, Y)=g(X, \phi Y) .
\end{gathered}
$$

An almost $r$-paracontact Riemannian manifold $M$ with structure $\sum=$ $\left(\phi, \xi_{\alpha}, \eta^{\alpha}, g\right)_{\alpha \in(r)}$ is said to be of $S$-paracontact type if [4]

$$
\Phi(X, Y)=\left(\nabla^{*}{ }_{Y} \eta^{\alpha}\right)(X), \alpha \in(r)
$$

for the Riemannian connection $\nabla^{*}$ on $M$. An almost $r$-paracontact Riemannian manifold $M$ with a structure $\sum=\left(\phi, \xi_{\alpha}, \eta^{\alpha}, g\right)_{\alpha \in(r)}$ is said to be of P-Sasakian 
type if it satisfies (2.6) and (2.7)

$$
\begin{aligned}
\left(\nabla^{*}{ }_{Z} \Phi\right)(X, Y)= & -\sum_{\alpha} \eta^{\alpha}(X)\left[g(Y, Z)-\sum_{\beta} \eta^{\beta}(Y) \eta^{\beta}(Z)\right] \\
& -\sum_{\alpha} \eta^{\alpha}(Y)\left[g(X, Z)-\sum_{\beta} \eta^{\beta}(X) \eta^{\beta}(Z)\right]
\end{aligned}
$$

for all vector fields $X, Y$ and $Z$ on $M$ [7]. The conditions (2.6) and (2.7) are equivalent respectively to

$$
\begin{gathered}
\phi X=\nabla_{X}^{*} \xi_{\alpha}, \alpha \in(r), \\
\left(\nabla_{Y}^{*} \phi\right)(X)=-\sum_{\alpha} \eta^{\alpha}(X)\left[Y-\eta^{\alpha}(Y) \xi_{\alpha}\right] \\
-\left[g(X, Y)-\sum_{\alpha} \eta^{\alpha}(X) \eta^{\alpha}(Y)\right] \sum_{\beta} \xi_{\beta} .
\end{gathered}
$$

A quarter symmetric metric connection $\nabla$ on $M$ is defined as

$$
\nabla_{\bar{X}} \bar{Y}=\nabla_{\bar{X}}^{*} \bar{Y}+\eta^{\alpha}(\bar{Y}) \phi \bar{X}-g(\phi \bar{X}, \bar{Y}) \xi_{\alpha}, \alpha \in(r) .
$$

Using (2.10) in (2.8) and (2.9), we get

$$
\begin{gathered}
\nabla_{X} \xi_{\alpha}=2 \phi X \\
\left(\nabla_{Y} \phi\right)(X)=-\sum_{\alpha} \eta^{\alpha}(X)\left[Y-\eta^{\alpha}(Y) \xi_{\alpha}\right] \\
-\left[g(X, Y)-\sum_{\alpha} \eta^{\alpha}(X) \eta^{\alpha}(Y)\right] \sum_{\beta} \xi_{\beta} \\
-g(X, Y) \xi_{\alpha}+\sum_{\alpha} \eta^{\alpha}(X) \eta^{\alpha}(Y) \xi_{\alpha}
\end{gathered}
$$

\section{Hypersurfaces of almost $r$-paracontact Riemannian manifold endowed with a quarter symmetric metric connection}

Let $M^{n+1}$ be an almost $r$-paracontact Riemannian manifold with a positive definite metric $g$ and $M^{n}$ be the hypersurface immersed in $M^{n+1}$ by the immersion $\tau: M^{n} \rightarrow M^{n+1}$. If $B$ denotes the differential of $\tau$, then any vector field $\bar{X} \in M^{n}$ implies $B \bar{X} \in M^{n+1}$. We denote the objects belonging to $M^{n}$ by the mark of hyphen placed over them, for example $\bar{\phi}, \bar{X}, \bar{\eta}, \bar{\xi}$. Let $N$ be the unit normal vector field to $M^{n}$. Then the induced metric $\bar{g}$ on $M^{n}$ is defined by

$$
\bar{g}(\bar{X}, \bar{Y})=g(\bar{X}, \bar{Y}) .
$$

Then we have [5]

$$
g(\bar{X}, N)=0, \quad g(N, N)=1 .
$$


If $\bar{\nabla}^{*}$ is the induced connection on hypersurface from $\nabla^{*}$ with respect to the unit normal vector $N$, then the Gauss formula is given by

$$
\nabla_{\bar{X}}^{*} \bar{Y}=\bar{\nabla}_{\bar{X}}^{*} \bar{Y}+h(\bar{X}, \bar{Y}) N,
$$

where $h$ is the second fundamental tensor satisfying

$$
h(\bar{Y}, \bar{X})=h(\bar{X}, \bar{Y})=\bar{g}(H \bar{X}, \bar{Y}) .
$$

If $\bar{\nabla}$ is the induced connection on hypersurface from $\nabla$ with respect to the unit normal vector $N$, then we have

$$
\nabla_{\bar{X}} \bar{Y}=\bar{\nabla}_{\bar{X}} \bar{Y}+m(\bar{X}, \bar{Y}) N,
$$

where $m$ is a tensor field of type $(0,2)$ of hypersurface $M^{n}$. From $(2.10)$, we obtain

$$
\nabla_{\bar{X}} \bar{Y}=\nabla_{\bar{X}}^{*} \bar{Y}+\eta^{\alpha}(\bar{Y})(\bar{\phi} \bar{X}+b(\bar{X}) N)-\bar{g}(\bar{\phi} \bar{X}, \bar{Y}) \xi_{\alpha},
$$

where $\phi \bar{X}=\bar{\phi} \bar{X}+b(\bar{X}) N$. From equations (3.3), (3.4) and (3.5), we get

$$
\begin{aligned}
\bar{\nabla}_{\bar{X}} \bar{Y}+m(\bar{X}, \bar{Y}) N= & \bar{\nabla}_{\bar{X}}^{*} \bar{Y}+h(\bar{X}, \bar{Y}) N+\eta^{\alpha}(\bar{Y}) \bar{\phi} \bar{X} \\
& +\bar{\eta}^{\alpha}(\bar{Y}) b(\bar{X}) N-\bar{g}(\bar{\phi} \bar{X}, \bar{Y})\left(\bar{\xi}_{\alpha}+a_{\alpha} N\right),
\end{aligned}
$$

where $\xi_{\alpha}=\bar{\xi}_{\alpha}+a_{\alpha} N$ and $\bar{\eta}^{\alpha}(\bar{X})=\eta^{\alpha}(\bar{X})$ for each $\alpha \in(r)$. By taking the tangential and normal parts from the both sides, we get respectively

$$
\begin{aligned}
\bar{\nabla}_{\bar{X}} \bar{Y} & =\bar{\nabla}_{\bar{X}}^{*} \bar{Y}+\bar{\eta}^{\alpha}(\bar{Y}) \bar{\phi} \bar{X}-\bar{g}(\bar{\phi} \bar{X}, \bar{Y}) \bar{\xi}_{\alpha}, \\
m(\bar{X}, \bar{Y}) & =h(\bar{X}, \bar{Y})+\bar{\eta}^{\alpha}(\bar{Y}) b(\bar{X})-a_{\alpha} \bar{g}(\bar{\phi} \bar{X}, \bar{Y}) .
\end{aligned}
$$

Thus we get the following theorem.

Theorem 3.1. The connection induced on a hypersurface of an almost $r$ paracontact Riemannian manifold endowed with a quarter symmetric metric connection with respect to the unit normal vector is also a quarter symmetric metric connection.

From (3.4) and (3.6), we have

$$
\nabla_{\bar{X}} \bar{Y}=\bar{\nabla}_{\bar{X}} \bar{Y}+\left\{h(\bar{X}, \bar{Y})-a_{\alpha} \bar{g}(\bar{\phi} \bar{X}, \bar{Y})+\bar{\eta}^{\alpha}(\bar{Y}) b(\bar{X})\right\} N,
$$

which is the Gauss formula for a quarter symmetric metric connection. The Weingarten formula with respect to the Riemannian connection $\nabla^{*}$ is given by

$$
\nabla_{\bar{X}}^{*} N=-H \bar{X}
$$

for every $\bar{X}$ in $M^{n}$, where $H$ is a tensor field of type $(1,1)$ of $M^{n}$ given by

$$
\bar{g}(H \bar{X}, \bar{Y})=h(\bar{X}, \bar{Y})=h(\bar{Y}, \bar{X}) .
$$

From equation (2.10), we have

$$
\nabla_{\bar{X}} N=\nabla_{\bar{X}}^{*} N+a_{\alpha} \bar{\phi} \bar{X}-b(\bar{X}) \bar{\xi}_{\alpha},
$$

where we have put

$$
\eta^{\alpha}(N)=a_{\alpha}=m\left(\xi_{\alpha}\right) .
$$


HYPERSURFACES OF ALMOST $r$-PARACONTACT RIEMANNIAN MANIFOLD 481 From (3.8) and (3.10), we have

$$
\nabla_{\bar{X}} N=-H \bar{X}+a_{\alpha} \bar{\phi} \bar{X}-b(\bar{X}) \bar{\xi}_{\alpha},
$$

which is the Weingarten formula with respect to the quarter symmetric metric connection.

Now, suppose that $\sum=\left(\phi, \xi_{\alpha}, \eta^{\alpha}, g\right)_{\alpha \in(r)}$ is an almost $r$-paracontact Riemannian structure on $M^{n+1}$. Then every vector field $X$ on $M^{n+1}$ is decomposed as

$$
X=\bar{X}+\lambda(X) N
$$

where $\lambda$ is an 1 -form on $M^{n+1}$ and $\bar{X}$ is any vector field and $N$ is normal vector on $M^{n}$. Also we have

$$
\begin{gathered}
\phi \bar{X}=\bar{\phi} \bar{X}+b(\bar{X}) N, \\
\phi N=\bar{N}+K N,
\end{gathered}
$$

where $\bar{\phi}$ is a tensor field of type $(1,1), b$ is an 1 -form and $K$ is a scalar function on $M^{n}$. For each $\alpha \in(r)$, we have

$$
\xi_{\alpha}=\bar{\xi}_{\alpha}+a_{\alpha} N
$$

where $a_{\alpha}=m\left(\xi_{\alpha}\right)=\eta^{\alpha}(N)$. Now, we define $\bar{\eta}^{\alpha}$ as

$$
\bar{\eta}^{\alpha}(\bar{X})=\eta^{\alpha}(\bar{X}), \alpha \in(r) .
$$

Making use of (3.13), (3.14), (3.15) and (3.11), we obtain from (2.1) through (2.5) for $\alpha \in(r)$

$$
\begin{gathered}
b(\bar{N})+K^{2}=1-\sum_{\alpha}\left(a_{\alpha}\right)^{2}, \\
K a_{\alpha}+b\left(\bar{\xi}_{\alpha}\right)=0, \\
\Phi(\bar{X}, \bar{Y})=\bar{g}(\bar{\phi} \bar{X}, \bar{Y})=\bar{g}(\bar{X}, \bar{\phi} \bar{Y})=\bar{\Phi}(\bar{X}, \bar{Y}) .
\end{gathered}
$$

Making use of (3.1), (3.2), (3.13), (3.14) and (2.5), we have

$$
g(\bar{\phi} \bar{X}, N)=g(\phi \bar{X}, N)-b(\bar{X})=g(\bar{X}, \phi N)-b(\bar{X})=0 .
$$

Hence we get

$$
g(\bar{X}, \bar{N})=b(\bar{X}) .
$$


Differentiating covariantly (3.13) and (3.14) along $M^{n}$ and making use of (3.7) and (3.12), we get respectively

$$
\begin{aligned}
\left(\nabla_{\bar{Y}} \phi\right) \bar{X}= & \left(\bar{\nabla}_{\bar{Y}} \bar{\phi}\right) \bar{X}-\left(h(\bar{X}, \bar{Y})-a_{\alpha} \bar{g}(\bar{\phi} \bar{Y}, \bar{X})+\bar{\eta}^{\alpha}(\bar{X}) b(\bar{Y})\right) \bar{N} \\
& +[(\bar{\nabla} \bar{Y} b)(\bar{X})+h(\bar{\phi} \bar{X}, \bar{Y})-(h(\bar{X}, \bar{Y}) \\
& \left.-a_{\alpha} \bar{g}(\bar{\phi} \bar{Y}, \bar{X})+\bar{\eta}^{\alpha}(\bar{X}) b(\bar{Y})\right) K-a_{\alpha} \bar{g}(\bar{X}, \bar{Y}) \\
& \left.+a_{\alpha} \sum_{\alpha} \bar{\eta}^{\alpha}(\bar{X}) \bar{\eta}^{\alpha}(\bar{Y})-2 a_{\alpha} b(\bar{X}) b(\bar{Y})\right] N \\
& -b(\bar{X})(H \bar{Y})-b(\bar{X}) b(\bar{Y}) \bar{\xi}_{\alpha}-a_{\alpha} b(\bar{X}) \bar{\phi} \bar{Y} \\
\left(\nabla_{\bar{Y}} \phi\right) N= & \bar{\nabla}_{\bar{Y}} \bar{N}+\left[\bar{Y}(K)+2\left(a_{\alpha}\right)^{2} \bar{\eta}^{\alpha}(\bar{Y})+h(\bar{X}, \bar{N})+b(H \bar{Y})\right] N \\
& +\bar{\phi}(H \bar{Y})-K(H \bar{Y})+a_{\alpha}\left(\bar{Y}-\bar{\eta}^{\alpha}(\bar{Y}) \bar{\xi}_{\alpha}\right) \\
& +K(\bar{\phi} \bar{Y})-K b(\bar{Y}) \bar{\xi}_{\alpha} .
\end{aligned}
$$

From (3.11) and (3.15), we have

$$
\begin{aligned}
\nabla_{\bar{Y}} \xi_{\alpha}= & \bar{\nabla}_{\bar{Y}} \bar{\xi}_{\alpha}-a_{\alpha}(H \bar{Y})+\left(a_{\alpha}\right)^{2} \bar{\phi} \bar{Y}-b(\bar{Y}) a_{\alpha} \bar{\xi}_{\alpha} \\
+\left[\bar{Y}\left(a_{\alpha}\right)+\right. & \left.h\left(\bar{Y}, \bar{\xi}_{\alpha}\right)+b(\bar{Y})-\left(a_{\alpha}\right)^{2} b(\bar{Y})-a_{\alpha} \bar{g}\left(\bar{\phi} \bar{Y}, \bar{\xi}_{\alpha}\right)\right] N \\
\left(\nabla_{\bar{Y}} \eta^{\alpha}\right)(\bar{X})= & \left(\bar{\nabla} \bar{Y}_{\bar{Y}} \bar{\eta}^{\alpha}\right)(\bar{X})-a_{\alpha} h(\bar{Y}, \bar{X}) \\
& -a_{\alpha} \bar{\eta}^{\alpha}(\bar{X}) b(\bar{Y})+\left(a_{\alpha}\right)^{2} \bar{g}(\bar{\phi} \bar{Y}, \bar{X}) .
\end{aligned}
$$

From the identity $\left(\nabla_{Z} \Phi\right)(X, Y)=g\left(\left(\nabla_{Z} \phi\right)(X), Y\right)$, making use of (3.19), (3.20) and (3.21), we have

$$
\begin{aligned}
\left(\nabla_{\bar{Z}} \Phi\right)(\bar{X}, \bar{Y})= & \left(\bar{\nabla}_{\bar{Z}} \bar{\Phi}\right)(\bar{X}, \bar{Y})-b(\bar{X}) h(\bar{Z}, \bar{Y})-b(\bar{Y}) h(\bar{Z}, \bar{X}) \\
& +a_{\alpha} b(\bar{X}) \bar{\Phi}(\bar{Y}, \bar{Z})+a_{\alpha} b(\bar{Y}) \bar{\Phi}(\bar{X}, \bar{Z}) \\
& -b(\bar{X}) b(\bar{Z}) \bar{\eta}^{\alpha}(\bar{Y})-b(\bar{Y}) b(\bar{Z}) \bar{\eta}^{\alpha}(\bar{X}) .
\end{aligned}
$$

From the above identities, we have the followings.

Theorem 3.2. If $M^{n}$ is an invariant hypersurface immersed in an almost $r$-paracontact Riemannian manifold $M^{n+1}$ endowed with a quarter symmetric metric connection with structure $\sum=\left(\phi, \xi_{\alpha}, \eta^{\alpha}, g\right)_{\alpha \in(r)}$, then either

(i) All $\xi_{\alpha}$ are tangent to $M^{n}$ and $M^{n}$ admits an almost r-paracontact Riemannian structure $\sum_{1}=\left(\bar{\phi}, \bar{\xi}_{\alpha}, \bar{\eta}^{\alpha}, \bar{g}\right)_{\alpha \in(r)}(n-r>2)$ or

(ii) One of $\xi_{\alpha}\left(\right.$ say, $\left.\xi_{r}\right)$ is normal to $M^{n}$ and remaining $\xi_{\alpha}$ are tangent to $M^{n}$ and $M^{n}$ admits an almost $(r-1)$-paracontact Riemannian structure $\sum_{2}=\left(\bar{\phi}, \bar{\xi}_{i}, \bar{\eta}^{i}, \bar{g}\right)_{i \in(r)}(n-r>1)$.

Proof. From (3.18), $K a_{\alpha}=0, \alpha \in(r)$. Hence we have the two possibilities when $K=0$ or $K \neq 0$.

(i) If $K \neq 0$, then $a_{\alpha}=0$ and $\xi_{\alpha}=\bar{\xi}_{\alpha}$ (all $\xi_{\alpha}$ are tangent to $M^{n}$ ) and the structure $\left(\bar{\phi}, \bar{\xi}_{\alpha}, \bar{\eta}^{\alpha}, \bar{g}\right)_{\alpha \in(r)}$ is an almost $r$-paracontact Riemannian structure on $M^{n}$ 
(ii) If $K=0$, then $\phi(N)=0$. Let $N=\xi_{r}$, then $\bar{\xi}_{r}=0, a_{r}=1, \bar{\eta}^{r}=0$. From (3.17) $\sum_{\alpha}\left(a_{\alpha}\right)^{2}=1$ and since $a_{r}=1, \sum_{i}\left(a_{i}\right)^{2}=0, i \in(r-1)$. Thus $a_{i}=0$ for all $i \in(r-1)$. Thus, $\xi_{i}=\bar{\xi}_{i}, \xi_{r}=N\left(\right.$ all $\xi_{\alpha}$ but one tangent to $\left.M^{n}\right)$. Hence structure $\left(\bar{\phi}, \bar{\xi}_{i}, \bar{\eta}^{i}, \bar{g}\right)_{i \in(r-1)}$ is an almost $(r-1)$-paracontact structure on $M^{n}$.

Corollary 3.1. If $M^{n}$ is a hypersurface immersed in an almost $r$-paracontact Riemannian manifold $M^{n+1}$ with a structure $\sum=\left(\phi, \xi_{\alpha}, \eta^{\alpha}, g\right)_{\alpha \in(r)}$ endowed with a quarter symmetric metric connection, then the following statements are equivalent:

(a) $M^{n}$ is invariant.

(b) The Normal field $N$ is an eigenvector of $\phi$.

(c) All $\xi_{\alpha}$ are tangent to $M^{n}$ if and only if $M^{n}$ admits an almost $r$ paracontact Riemannian structure $\sum_{1}$, or one of $\xi_{\alpha}$ is normal and $(r-1)$ remaining $\xi_{i}$ are tangent to $M^{n}$ if and only if $M^{n}$ admits an almost $(r-1)$-paracontact Riemannian structure $\sum_{2}$.

Theorem 3.3. If $M^{n}$ is an invariant hypersurface immersed in an almost $r$ paracontact Riemannian manifold of P-Sasakian type endowed with a quarter symmetric metric connection, then the induced almost $r$-paracontact Riemannian structure $\sum_{1}$ or $(r-1)$-paracontact Riemannian structure $\sum_{2}$ are also of $P$-Sasakian type.

Proof. Making use of (3.1), (3.16), (3.19), (3.24) and (3.25), we observe that the conditions (2.11) and (2.12) are satisfied for both $\sum_{1}$ and $\sum_{2}$.

Lemma 3.1. $\bar{\nabla}_{\bar{X}}(\operatorname{trace} \bar{\phi})=\operatorname{trace}\left(\bar{\nabla}_{\bar{X}} \bar{\phi}\right)$.

Proof. Let $\left\{e_{1}, e_{2}, e_{3}, \ldots, e_{n}\right\}$ be an orthogonal basis of $T M^{n}$, then trace $\bar{\phi}=$ $\sum_{a} \bar{g}\left(\bar{\phi}\left(e_{a}, e_{a}\right)\right)$ for $a \in(n-1)$. Let $\bar{\nabla}_{\bar{X}} e_{a}=A_{a}^{b} e_{b}$ and $\phi\left(e_{a}\right)=B_{a}^{b} e_{b}$, then from $0=\bar{g}\left(\bar{\nabla}_{\bar{X}} e_{a}, e_{b}\right)+\bar{g}\left(e_{a}, \bar{\nabla}_{\bar{X}} e_{b}\right)$ and from $\bar{g}\left(\bar{\phi}\left(e_{a}\right), e_{b}\right)=\bar{g}\left(e_{a}, \bar{\phi}\left(e_{b}\right)\right)$, we obtain $A_{a}^{b}-A_{b}^{a}=0$ and $B_{b}^{a}=B_{a}^{b}$. Hence $\sum_{\alpha} \bar{g}\left(\bar{\phi}\left(e_{a}\right), \bar{\nabla}_{\bar{X}} e_{a}\right)=\sum_{a, b} A_{b}^{a} B_{b}^{a}=0$ and we have

$$
\bar{\nabla}_{\bar{X}}(\operatorname{trace} \bar{\phi})=\sum_{a} \bar{g}\left(\left(\bar{\nabla}_{\bar{X}} \bar{\phi}\right)\left(e_{a}\right), e_{a}\right)+2 \sum_{a} \bar{g}\left(\bar{\phi}\left(e_{a}\right), \bar{\nabla}_{\bar{X}} e_{a}\right)=\operatorname{trace}\left(\bar{\nabla}_{\bar{X}} \bar{\phi}\right)
$$

Theorem 3.4. Let $M^{n}$ be a non-invariant hypersurface of an almost r-paracontact Riemannian manifold $M^{n+1}$ endowed with a quarter symmetric metric connection with a structure $\sum=\left(\phi, \xi_{\alpha}, \eta^{\alpha}, g\right)_{\alpha \in(r)}$ satisfying $\nabla \phi=0$ along $M^{n}$, then $M^{n}$ is totally geodesic if and only if

$$
\left(\bar{\nabla}_{\bar{Y}} \bar{\phi}\right) \bar{X}+a_{\alpha} \bar{g}(\bar{\phi} \bar{Y}, \bar{X}) \bar{N}+a_{\alpha} b(\bar{X}) \bar{\phi} \bar{Y}-b(\bar{X}) b(\bar{Y}) \bar{\xi}_{\alpha}+\bar{\eta}^{\alpha}(\bar{X}) b(\bar{Y}) \bar{N}=0 .
$$


Proof. From (3.21) we have

$$
\begin{aligned}
\left(\bar{\nabla}_{\bar{Y}} \phi\right) \bar{X}= & \left(\bar{\nabla}_{\bar{Y}} \bar{\phi}\right) \bar{X}-\left(h(\bar{X}, \bar{Y})-a_{\alpha} \bar{g}(\bar{\phi} \bar{Y}, \bar{X})+\bar{\eta}^{\alpha}(\bar{X}) b(\bar{Y})\right) \bar{N} \\
& +\left[\left(\bar{\nabla}_{\bar{Y}} b\right)(\bar{X})+h(\bar{\phi} \bar{X}, \bar{Y})-(h(\bar{X}, \bar{Y})\right. \\
& \left.-a_{\alpha} \bar{g}(\bar{\phi} \bar{Y}, \bar{X})+\bar{\eta}^{\alpha}(\bar{X}) b(\bar{Y})\right) K \\
& \left.-a_{\alpha} \bar{g}(\bar{X}, \bar{Y})+a_{\alpha} \sum_{\alpha} \bar{\eta}^{\alpha}(\bar{X}) \bar{\eta}^{\alpha}(\bar{Y})-2 a_{\alpha} b(\bar{X}) b(\bar{Y})\right] N \\
& -b(\bar{X})(H \bar{Y})-b(\bar{X}) b(\bar{Y}) \bar{\xi}_{\alpha}+b(\bar{X}) a_{\alpha} \bar{\phi} \bar{Y}
\end{aligned}
$$

If $M^{n}$ is totally geodesic, then $h=0$ and $H=0$. Thus from (3.26), we get

$\left(\bar{\nabla}_{\bar{Y}} \phi\right) \bar{X}+a_{\alpha} \bar{g}(\bar{\phi} \bar{Y}, \bar{X}) \bar{N}+a_{\alpha} b(\bar{X}) \bar{\phi} \bar{Y}-b(\bar{X}) b(\bar{Y}) \bar{\xi}_{\alpha}+\bar{\eta}^{\alpha}(\bar{X}) b(\bar{Y}) \bar{N}=0$.

Conversely, if

$$
\left(\bar{\nabla}_{\bar{Y}} \phi\right) \bar{X}+a_{\alpha} \bar{g}(\bar{\phi} \bar{Y}, \bar{X}) \bar{N}+a_{\alpha} b(\bar{X}) \bar{\phi} \bar{Y}-b(\bar{X}) b(\bar{Y}) \bar{\xi}_{\alpha}+\bar{\eta}^{\alpha}(\bar{X}) b(\bar{Y}) \bar{N}=0
$$

then it holds

$$
h(\bar{Y}, \bar{X}) \bar{N}+b(\bar{X}) H(\bar{Y})=0 .
$$

Making use of (3.9) and (3.20), we have

$$
h(\bar{X}, \bar{Y}) b(\bar{Z})+h(\bar{X}, \bar{Z}) b(\bar{Y})=0 .
$$

Using (3.27), we get from (3.9)

$$
h(\bar{X}, \bar{Z}) b(\bar{Y})=h(\bar{X}, \bar{Y}) b(\bar{Z}) .
$$

From (3.28) and (3.29), we get $b(\bar{Z}) h(\bar{X}, \bar{Y})=0$ which gives $h=0$ as $b \neq 0$. Using $h=0$ in (3.27), we get $H=0$. Thus $h=0$ and $H=0$. Hence $M^{n}$ is totally geodesic.

Theorem 3.5. Let $M^{n}$ be a non-invariant hypersurface of an almost r-paracontact Riemannian manifold $M^{n+1}$ endowed with a quarter symmetric metric connection satisfying $\nabla \phi=0$ along $M^{n}$ and if trace $\bar{\phi}=$ constant, then

$$
h(\bar{X}, \bar{N})=\sum_{a}\left[a_{\alpha} b\left(e_{a}\right) \Phi\left(e_{a}, \bar{X}\right)-b(\bar{X}) b\left(e_{a}\right) \bar{\eta}^{\alpha}\left(e_{a}\right)\right],
$$

where $\bar{N}=\sum_{a} b\left(e_{a}\right) e_{a}$.

Proof. From (3.26) we have

$$
\bar{g}\left(\left(\bar{\nabla}_{\bar{Y}} \bar{\phi}\right) \bar{X}, \bar{X}\right)=2 b(\bar{X}) h(\bar{X}, \bar{Y})-2 a_{\alpha} b(\bar{X}) \bar{\Phi}(\bar{X}, \bar{Y})+2 b(\bar{X}) b(\bar{Y}) \bar{\eta}^{\alpha}(\bar{X})
$$

and

$$
\bar{\nabla}_{\bar{X}}(\operatorname{trace} \bar{\phi})=2 h(\bar{X}, \bar{N})-2 a_{\alpha} \sum_{a} b\left(e_{a}\right) z \bar{\Phi}\left(e_{a}, \bar{X}\right)+2 \sum_{a} b(\bar{X}) b\left(e_{a}\right) \bar{\eta}^{\alpha}\left(e_{a}\right)
$$

Using Lemma 3.1, we get

$$
h(\bar{X}, \bar{N})=\sum_{a}\left[a_{\alpha} b\left(e_{a}\right) \bar{\Phi}\left(e_{a}, \bar{X}\right)-b(\bar{X}) b\left(e_{a}\right) \bar{\eta}^{\alpha}\left(e_{a}\right)\right],
$$

where $\bar{N}=\sum_{a} b\left(e_{a}\right) e_{a}$. 
Let $M^{n}$ be an almost $r$-paracontact Riemannian manifold of $S$-paracontact type with a quarter symmetric metric connection, then from (2.11), (3.13) and (3.23), we get

$$
\bar{\phi} \bar{X}=\frac{1}{2}\left[\bar{\nabla}_{\bar{X}} \bar{\xi}_{\alpha}-a_{\alpha}(H \bar{X})+\left(a_{\alpha}\right)^{2} \bar{\phi}(\bar{X})-a_{\alpha} b(\bar{X}) \bar{\xi}_{\alpha}\right], \alpha \in(r),
$$

$$
b(\bar{X})=\frac{1}{2}\left[\bar{X}\left(a_{\alpha}\right)+h\left(\bar{X}, \bar{\xi}_{\alpha}\right)+\left(1-\left(a_{\alpha}\right)^{2}\right) b(\bar{X})-a_{\alpha} \bar{g}\left(\bar{\phi} \bar{X}, \bar{\xi}_{\alpha}\right)\right], \alpha \in(r)
$$

Making use of (3.31), we have that if $M^{n}$ is totally geodesic, then $a_{\alpha}=0$ and $h=0$. Hence $b=0$, that is, $M^{n}$ is invariant. Thus we have the following.

Proposition 3.1. If $M^{n}$ is totally geodesic hypersurface of an almost r-paracontact Riemannian manifold $M^{n+1}$ endowed with a quarter symmetric metric connection of $S$-paracontact type with a structure $\sum=\left(\phi, \xi_{\alpha}, \eta^{\alpha}, g\right)_{\alpha \in(r)}$ and all $\xi_{\alpha}$ are tangent to $M^{n}$, then $M^{n}$ is invariant.

Theorem 3.6. If $M^{n}$ is an anti-invariant hypersurface of an almost r-paracontact Riemannian manifold $M^{n+1}$ endowed with a quarter symmetric metric connection of $S$-paracontact type with a structure $\sum=\left(\phi, \xi_{\alpha}, \eta^{\alpha}, g\right)_{\alpha \in(r)}$, then all $\bar{\xi}_{\alpha}$ are parallel to $M^{n}$.

Proof. If $M^{n}$ is anti-invariant, then $\bar{\phi}=0$ and $a_{\alpha}=0$ and from (3.30) we have $\bar{\nabla}_{\bar{X}} \bar{\xi}_{\alpha}=0$.

Now, let $M^{n}$ be an almost $r$-paracontact Riemannian manifold of $P$-Sasakian type endowed with a quarter symmetric metric connection. Then from (2.12) and (3.21), we have

$$
\begin{aligned}
& (\bar{\nabla} \overline{\bar{Y}} \bar{\phi}) \bar{X}-\left[h(\bar{X}, \bar{Y})-a_{\alpha} \bar{g}(\bar{\phi} \bar{Y}, \bar{X})+\bar{\eta}^{\alpha}(\bar{X}) b(\bar{Y})\right] \bar{N} \\
& -b(\bar{X})(H \bar{Y})+a_{\alpha} b(\bar{X}) \bar{\phi} \bar{Y}-b(\bar{X}) b(\bar{Y}) \bar{\xi}_{\alpha} \\
= & -\sum_{\alpha} \bar{\eta}^{\alpha}(\bar{X})\left(\bar{Y}-\bar{\eta}^{\alpha}(\bar{X}) \bar{\xi}_{\alpha}\right) \\
& -\left[\bar{g}(\bar{X}, \bar{Y})-\sum_{\alpha} \bar{\eta}^{\alpha}(\bar{X}) \bar{\eta}^{\alpha}(\bar{Y})\right] \sum_{\beta} \bar{\xi}_{\beta}-\bar{g}(\bar{X}, \bar{Y}) \bar{\xi}_{\alpha} \\
& +\sum_{\alpha} \bar{\eta}^{\alpha}(\bar{X}) \bar{\eta}^{\alpha}(\bar{Y}) \bar{\xi}_{\alpha} .
\end{aligned}
$$

Theorem 3.7. Let $M^{n+1}$ be an almost r-paracontact Riemannian manifold of $P$-Sasakian type endowed with a quarter symmetric metric connection with a structure $\sum=\left(\phi, \xi_{\alpha}, \eta^{\alpha}, g\right)_{\alpha \in(r)}$, and let $M^{n}$ be a hypersurface immersed in $M^{n+1}$ such that none of $\xi_{\alpha}$ are tangent to $M^{n}$. Then $M^{n}$ is totally geodesic if 
and only if

$$
\begin{aligned}
\left(\bar{\nabla}_{\bar{Y}} \bar{\phi}\right) \bar{X}= & -a_{\alpha} b(\bar{X}) \bar{\phi} \bar{Y}-a_{\alpha} \bar{g}(\bar{\phi} \bar{Y}, \bar{X}) \bar{N}-\sum_{\alpha} \bar{\eta}^{\alpha}(\bar{X})\left[\bar{Y}-\bar{\eta}^{\alpha}(\bar{Y}) \bar{\xi}_{\alpha}\right] \\
& +b(\bar{X}) b(\bar{Y}) \bar{\xi}_{\alpha}-\left[\bar{g}(\bar{X}, \bar{Y})-\sum_{\alpha} \bar{\eta}^{\alpha}(\bar{X}) \bar{\eta}^{\alpha}(\bar{Y})\right] \sum_{\beta} \bar{\xi}_{\beta} \\
& -\bar{g}(\bar{X}, \bar{Y}) \bar{\xi}_{\alpha}+\sum_{\alpha} \bar{\eta}^{\alpha}(\bar{X}) \bar{\eta}^{\alpha}(\bar{Y}) \bar{\xi}_{\alpha}
\end{aligned}
$$

Proof. If (3.33) is satisfied, then from (3.32), we get $h(\bar{X}, \bar{Y}) \bar{N}+b(\bar{X}) H(\bar{Y})=0$. Since $b \neq 0$, so that $h(\bar{X}, \bar{Y})=0$. Hence $M^{n}$ is totally geodesic. Conversely, let $M^{n}$ is totally geodesic, that is $H=0$, then from (3.32) we get (3.33) and from (3.31) we have $b=0$, which is contradiction. Hence $\xi_{\alpha}$ are not tangent to $M^{n}$.

\section{References}

1] T. Adati, Hypersurfaces of almost paracontact Riemannian manifolds, TRU Math. 17 (1981), no. 2, 189-198.

[2] M. Ahmad, C. Ozgur, and A. Haseeb, Hypersurfaces of almost $r$-paracontact Riemannian manifold endowed with a quarter symmetric non-metric connection, Accepted at Kyungpook Math. J.

[3] A. Bucki, Hypersurfaces of almost $r$-paracontact Riemannian manifolds, Tensor (N.S.) 48 (1989), no. 3, 245-251.

[4] A. Bucki and A. Miernowski, Invariant hypersurfaces of an almost r-paracontact manifold, Demonstratio Math. 19 (1986), no. 1, 113-121.

[5] B. Y. Chen, Geometry of Submanifolds, Pure and Applied Mathematics, No. 22. Marcel Dekker, Inc., New York, 1973.

[6] S. Golab, On semi-symmetric and quarter symmetric linear connections, Tensor (N.S.) 29 (1975), no. 3, 249-254.

[7] I. Mihai and K. Matsumoto, Submanifolds of an almost r-paracontact Riemannian manifold of P-Sasakian type, Tensor (N.S.) 48 (1989), no. 2, 136-142.

[8] R. S. Mishra and S. N. Pandey, On quarter symmetric metric F-connections, Tensor (N.S.) 34 (1980), no. 1, 1-7.

[9] S. C. Rastogi, On quarter symmetric metric connection, C. R. Acad. Bulgare Sci. 31 (1978), no. 7, 811-814.

[10] - On quarter symmetric metric connections, Tensor (N.S.) 44 (1987), no. 2, $133-141$.

Mobin AHMAD

Department of Mathematics

INTEGRAL UNIVERSITY

Kursi-RoAD, LUCKNOW, 226026, INDIA

E-mail address: mobinahmad@rediffmail.com

JAE-BOK JUN

Department of Mathematics

College of Natural Science

KOOKMIN UNIVERSITY

SEOUL 136-702, KoreA

E-mail address: jbjun@kookmin.ac.kr 
HYPERSURFACES OF ALMOST $r$-PARACONTACT RIEMANNIAN MANIFOLD 487

Abdul Haseeb

Department of Mathematics

INTEGRAL UNIVERSITY

KuRsi-RoAd, LuCKNOW, 226026, INDIA

E-mail address: malik_haseeb@indiatimes.com 